\title{
Do halos exist on the dripline of deformed nuclei?
}

\author{
F.M. Nunes, B. Avez, T. Duguet \\ National Superconducting Cyclotron Laboratory and Department of Physics and Astronomy, \\ Michigan State University, East-Lansing MI 48824-1321
}

\begin{abstract}
A study of the effect of deformation and pairing on the development of halo nuclei is presented. Exploratory three-body core $+n+n$ calculations show that both the NN interaction and the deformation/excitation of the core hinder the formation of the halo. Preliminary selfconsistent mean-field calculations are used to search for regions in the nuclear chart where halos could potentially develop. These are also briefly discussed.
\end{abstract}

\section{MOTIVATION}

During the early years of Radioactive Nuclear Beam physics, while the dripline for light nuclei was being explored, nuclear halos became a very hot topic [1]. Typical examples of halo nuclei include ${ }^{6} \mathrm{He},{ }^{11} \mathrm{Li},{ }^{14} \mathrm{Be}$ and ${ }^{19} \mathrm{C}$, all in the low mass region of the nuclear chart. The big open question, when moving toward heavier systems, is whether halos can develop when for instance $A>40$.

In order to successfully describe a halo nucleus, the structure model needs to take into account [3]: i) the very low density region in which the halo nucleons move, subject to an interaction that is closer to the free NN interaction than the realistic in-medium nuclear interaction; ii) the long tails of the wavefunctions and correct asymptotics of these tails, which contribute decisively to many nuclear properties; iii) the few-body dynamics of the valence nucleons relative to the core and between themselves. For these reasons, it is acceptable to decouple the halo degrees of freedom from the core's, simplifying the standard microscopic treatment: this is the basis for applying few-body models to light nuclear halos.

In heavier systems, the decoupling of core and valence nucleons may not be as straightforward. Mean-field studies in the past decades have shown that pairing is important, and in some regions of the nuclear chart (namely the deformed region), deformation is also necessary. As matter densities in Hartree Fock Bogolyubov (HFB) have a faster radial decay when pairing is included, one expects the pairing force to act against the halo formation. The pairing anti-halo effect was shown in Ref. [5] through selfconsistent Hartree Fock Bogolyubov calculations for Carbon isotopes. In opposition to [5], the results of Hamamoto et al. [6, 7] indicate that when an $s_{1 / 2}$ neutron approaches the dripline, it becomes decoupled from the mean field, which means they are less paired and consequently may give rise to a halo. One can then claim that, if the neutron becomes decoupled from the mean field, the HFB theory should not be used. Unfortunately, the calculations in [6, 7] are not self-consistent; the mean field is reduced arbitrarily, from a well bounded situation, to force the system toward the dripline. 
If the situation with pairing is controversial, the effect of deformation appears to be well settled. Nilsson model calculations performed by Hamamoto [8] suggest that $J_{z}=1 / 2^{+}$states $\left(J_{z}\right.$ being the projection of the angular momentum on the deformation axis), which contain s-wave components of the valence particle as well as other components with higher partial waves, become pure s-waves when the system it forced toward threshold. Thus, it is concluded that deformation does not hinder the halo formation [8].

This exotic feature is not solely associated to nuclear physics: halo manifestations appear also in atomic, molecular or condensed matter physics. A detailed review [2] compiles the results across the nuclear border, and develops a halo signature that removes the specific scale (nuclear, atomic, etc).

\section{RESULTS WITH THREE-BODY MODEL}

In Ref. [4] we study the structure of the ground state of a nucleus with two valence nucleons as the system approaches the two particle threshold. A three-body model of core $+n+n$ is used where the core is deformed and allowed to excite. The Faddeev Equations are solved within the hyperspherical method. Our starting point is the ${ }^{12} \mathrm{Be}$ model [9]. An important ingredient of the model [9] is the core $+n$ interaction fitted to reproduce the properties of ${ }^{11} \mathrm{Be}$. We artificially decrease this core $-n$ interaction,

$$
V_{n-\text { core }}(\vec{r})=\lambda V_{n-\text { core }}^{\text {be } 12}(\vec{r}) \text {. }
$$

with $\lambda \rightarrow 0$ to simulate the approach to the neutron dripline [4].

The correspondence from a few-body language to the mean-field terminology appropriate for heavy systems, is far from trivial. Nevertheless, in some way, pairing and deformation are included in this three-body model. In the limit of a very heavy core, the core-n interaction plays the role of the mean field in the microscopic description. The $\mathrm{NN}$ interaction $V_{n n}$ included in the three-body model would then be related to the pairing associated with the valence pair only, in the mean-field language. However the pairing force and $V_{n n}$ are not identical. Here $V_{n n}$ is a sum of gaussians fitted to the low energy $\mathrm{NN}$ phases shifts, containing central $\mathrm{s}, \mathrm{p}$ and $\mathrm{d}$ terms, as well as a spin orbit and a tensor force, while pairing in a typical mean-field calculation is zero range and s-wave only (see [4, 10] for a further discussion).

The hyperspherical expansion introduces a new quantum number $K$ related to the sum of the angular momenta associated with the Jacobi coordinates $(\mathrm{x}, \mathrm{y})[9]: K=$ $\left(l_{x}+l_{y}\right) / 2$. The resulting coupled channel equations contain a centrifugal barrier of the form $(K+3 / 2)(K+5 / 2) / \rho^{2}$, where $\rho=\sqrt{x^{2}+y^{2}}$ is the size of the halo. Even for the lowest hyper momentum $K=0$, there is a barrier reducing the halo effect. For a halo to appear, the $\mathrm{K}=0$ component needs to be the dominant component ( $>50 \%)$ [2]. Thus, we look at the probability of $\mathrm{K}=0$ in the ground state wavefunction as a function of the threebody binding energy (Fig. 1). The probabilities for the lowest hyperspherical harmonic component $(\mathrm{K}=0)$ as a function of the two-neutron separation energy are shown for four different cases: i) the case where a realistic $\mathrm{NN}$ interaction is included, as well as a quadrupole deformation $\beta_{2}=0.67$ for the ${ }^{10} \mathrm{Be}$ core that reproduces the experimental $B\left(E 2 ; 0^{+} \rightarrow 2^{+}\right)$; ii) the case where only the NN interaction is switched off; iii) the case 


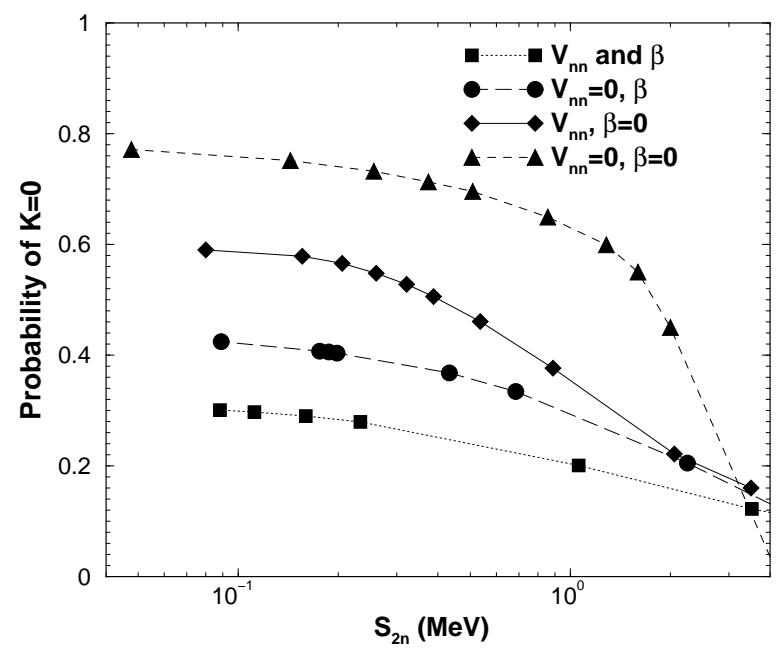

FIGURE 1. Probabilities for the $\mathrm{K}=0$ component in the ground state wavefunction of a ${ }^{12} \mathrm{Be}$-like system as a function of the two valence neutron separation energy.

where deformation/excitation is neglected but the NN interaction is included; iv) the case where both the NN interaction and core deformation/excitation are neglected.

The first thing that should be noted is that, even for no deformation/excitation and for no NN interaction, the system never develops a pure $\mathrm{K}=0$ state in the limit of zero binding. This result is associated with the fact that we are treating the system as a threebody system and it is in contrast with what would be obtained in the two body case [2]. Secondly, both the NN interaction and collective core degrees of freedom decrease considerably the limiting value for $\operatorname{Prob}(\mathrm{K}=0)$ when $S_{2 n} \rightarrow 0$. Ultimately, when both effects are included, the probability is well below $50 \%$, suggesting that no halo will appear.

Note that the physical input for this exploratory study was provided by ${ }^{12} \mathrm{Be}$. One can repeat the calculations reducing the deformation parameter and increasing the mass of the core, to simulate a heavier system more realistically [4]. However the model would still be inconsistent, in the sense that the core-n interaction would not be determined from the physical subsystem, and the approach to the dripline would remain artificial.

\section{PRELIMINARY HFB RESULTS}

The appropriate way to address the problem for medium mass to heavy nuclei is through a self-consistent model that contains both pairing and deformation [11]. Let us first concentrate on pairing only. A systematic HFB study was performed, searching for nuclei with the $3 s_{1 / 2}$ neutron orbital intercepting the Fermi level in the limit of stability [12]. All calculations were performed with the HFB code written in a 3D mesh [13]. We found that the $\mathrm{Cr}$ isotopes satisfied this condition. Using SLy4 [14] for the particle-hole channel and ULB zero-range density-dependent pairing [15] for the particle-particle channel, the neutron dripline is reached for ${ }^{80} \mathrm{Cr}$. We plot the proton and neutron Helm 
radii [16] and geometrical radii (Fig. 2) as a function of isotopic mass. The large difference between the neutron and proton Helm radii indicate a neutron skin. A neutron halo exists when there is a large difference between the neutron Helm and geometrical radii. We see that both are present on the $\mathrm{Cr}$ dripline. A detailed analysis of our results do not confirm those from Ref. [6] concerning the decoupling of the s-wave orbital from the core, when reaching the dripline [12].

Helm criteria

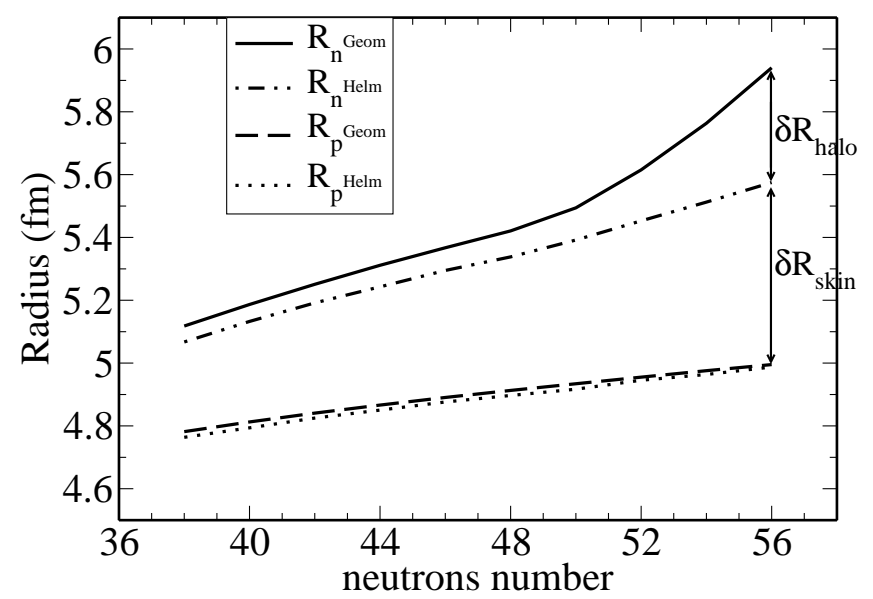

FIGURE 2. Helm criteria for halos and skins: geometrical and Helm radii for both neutrons and protons in $\mathrm{Cr}$ isotopes.

Helm criteria, $\mathrm{z}$ axis

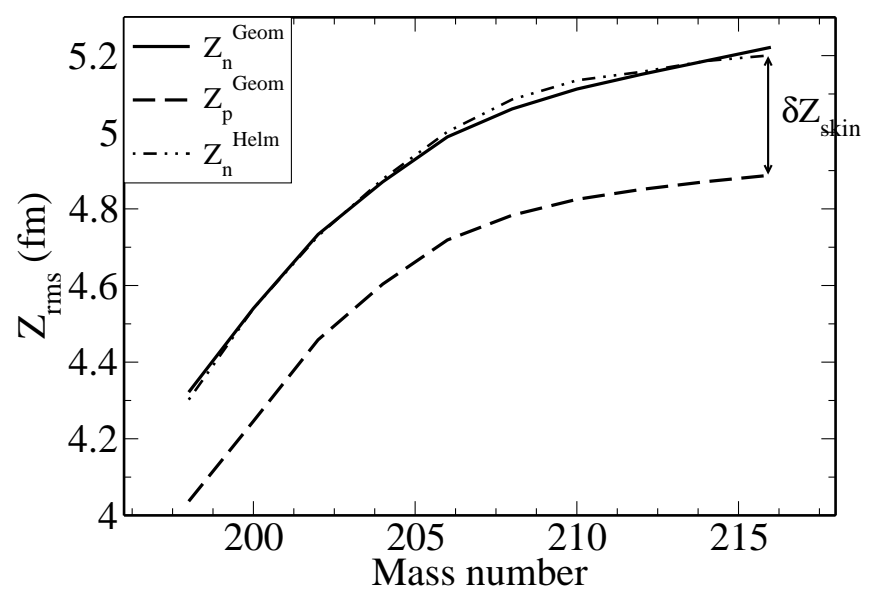

FIGURE 3. Same as Fig.(2) for Dy isotopes along the deformation axis.

Similar searches where performed in the region of deformed nuclei using HFB with even multipole deformation [17]. For the deformed region the orbital we need to concentrate on is the harmonic oscillator $1 / 2^{+}$Nilsson orbital coming from the $4 s_{1 / 2}$ shell in the limit of sphericity. Only for the prolate Dy isotopes did we find this valence neutron orbital to be close to the Fermi level at the dripline. The analysis based on the Helm and 
geometrical radii was performed on the Dy isotopes (see Fig. 3), similarly to the Cr case. For that purpose, an extension of the Helm criterion to deformed systems was proposed [12]. As there is no significant difference between the neutron's Helm and geometrical radii, we expect no halo to develop. Note that Dy isotopes demonstrate another minimum for oblate shape, which means that the correct ground state should have configuration admixture. Such a calculation was not performed due to its complexity, yet we do not expect it would reverse the result concerning halos.

From these preliminary self-consistent HFB studies we can draw the following conclusions:

- In a realistic situation, pairing does indeed oppose the development of the halo. This confirms the results of [5] and the three-body model [4]. The effect is not sufficiently strong to hinder completely a halo in ${ }^{80} \mathrm{Cr}$.

- The self-consistent HFB results do not show the decoupling of the neutron from the mean field seen by [6].

- Deformation in heavy nuclei seem to strongly hinder the appearance of a halo, as suggested by the three-body model [4] and in disagreement with [8]. No halos were found in the deformed region of the nuclear chart. In particular, the fully selfconsistent calculations do not confirm the purification of the $J_{z}=1 / 2^{+}$orbitals at the Fermi level into s-waves when approaching the dripline.

An article containing all details of these HFB studies will become available soon. As part of our near future plans, we will study the dependence on the choice of the microscopic pairing force, namely a better low-density behaviour and finite-range effect [10].

* This work has been partially supported by National Superconducting Cyclotron Laboratory at Michigan State University and the National Science Foundation under grants PHY-0456656 and PHY-0456903.

\section{REFERENCES}

1. P.G. Hansen, New Scientist, pg 38 October 1993.

2. A.S. Jensen, K. Riisager, D.V. Fedorov and E. Garrido, Rev. Mod. Phys. 76 (2004) 215.

3. F.M. Nunes, C.R. Physique 4 (2003) 489.

4. F.M. Nunes, Nucl. Phys. A 757 (2005) 349.

5. K. Bennaceur, J. Dobaczewski, M. Ploszajczak, Phys. Lett. B 496 (2000) 154.

6. I. Hamamoto and B.R. Mottelson, Phys. Rev. C 68 (2003) 034312.

7. I. Hamamoto and B.R. Mottelson, Phys. Rev. C 69 (2004) 064302.

8. I. Hamamoto, Phys. Rev. C 69 (2004) 041306.

9. F.M. Nunes et al., Nucl. Phys. A 609 (1996) 43.

10. T. Duguet, Phys. Rev. C 69 (2004 054317.

11. M. Bender, P.-H. Heenen, and P.-G. Reinhard, Rev. Mod. Phys. 75 (2003) 121.

12. Benoit Avez, Report of Summer Training on Halo Effect on Heavy nuclei NSCL, August 2005.

13. B. Gall et al., Z. Phys. A 348 (1994) 183.

14. E. Chabanat et al., Nucl. Phys. A 627 (1997) 710.

15. C. Rigollet and P. Bonche and H. Flocard and P.-H. Heenen, Phys. Rev. C (1999) 3120.

16. R. Helm, Phys. Rev. 104 (1956) 1466.

17. M. Bender, P. Bonche, T. Duguet, and P.-H. Heenen, Phys. Rev. C 69 (2004) 064303. 